\title{
Photons with sub-Planckian energy cannot efficiently probe space-time foam
}

\author{
Yanbei Chen, ${ }^{1, *}$ Linqing Wen, ${ }^{2, \dagger}$ and Yiqiu $\mathrm{Ma}^{2, *}$ \\ ${ }^{1}$ Theoretical Astrophysics 350-17, California Institute of Technology, Pasadena, California 91125, USA \\ ${ }^{2}$ Australian International Gravitational Research Centre, School of Physics, \\ University of Western Australia, 35 Stirling Hwy, Crawley, \\ Western Australia 6009, Australia
}

(Received 23 October 2013; published 24 September 2014)

\begin{abstract}
Extra-galactic sources of photons have been used to constrain space-time quantum fluctuations in the Universe. In these proposals, the fundamental "fuzziness" of distance caused by space-time quantum fluctuations has been directly identified with fluctuations in optical paths. Phase-front corrugations deduced from these optical-path fluctuations are then applied to light from extra-galactic point sources, and used to constrain various models of quantum gravity. However, when a photon propagates in three spatial dimensions, it does not follow a specific ray, but rather samples a finite, three-dimensional region around that ray - thereby averaging over space-time quantum fluctuations all through that region. We use a simple, random-walk type model to demonstrate that, once the appropriate wave optics is applied, the averaging of neighboring space-time fluctuations will cause much less distortion to the phase front. In our model, the extra suppression factor due to diffraction is the wave length in units of the Planck length, which is at least $10^{29}$ for astronomical observations.
\end{abstract}

DOI: 10.1103/PhysRevD.90.063011

PACS numbers: 95.75.Mn, 03.67.Lx, 04.60.-m

\section{INTRODUCTION}

There have been several proposals to use extragalactic point sources to constrain the quantum fluctuations in space-time [1-4]. It was argued that, space-time fluctuations cause random phase shifts of photons, and that these shifts accumulate throughout the very long light propagation path from the point source to the earth, causing wavefront distortion from a perfect spherical shape upon arrival at the earth. The manner in which these fluctuations accumulate depends on the specific model of quantum gravity phenomenology; and in particular, it has been claimed that the random walk model could be ruled out by existing imaging data from the Hubble Space Telescope. In this paper, we point out a serious omission in the theory so far employed by all such proposals, and argue that the random walk model, once given a closer look, cannot be ruled out at all by current or any foreseeable observations of extra galactic sources of photons.

In Refs. [1-4], based on the argument about the quantum space-time on the phenomenological level [1,5], the authors assumed photons originating from a point source to undergo a random phase shift $\Delta \phi$ due to space-time fluctuations:

$$
\Delta \phi \sim 2 \pi\left(l_{\mathrm{P}} / \lambda\right)^{\alpha}(L / \lambda)^{1-\alpha},
$$

where $l_{\mathrm{P}}$ is the Planck length, $\lambda$ is the wavelength of the light, $L$ is the light propagation length, and $\alpha$ is a parameter

\footnotetext{
*yanbei@its.caltech.edu

linqing.wen@uwa.edu.au

¥myqphy@gmail.com
}

that depends on specific models of quantum space-time phenomenology. In particular, $\alpha=1 / 2$ corresponds to the so-called random-walk model, which can be understood as having the speed of light fluctuating dramatically at the scale of $l_{\mathrm{P}}$. The relation (1) stems from phenomenological description of the quantumness of space-time by assuming that the uncertainty in distance measurement $\delta L$ due to space-time fluctuation over a distance $L$ is given by

$$
\delta L \geq L^{1-\alpha} l_{\mathrm{P}}^{\alpha}
$$

which was first derived by $\mathrm{Ng}$ and van Dam and then discussed by others $[4,6-8]$. Note that Eq. (1) is related to Eq. (2) by $\Delta \phi \sim 2 \pi(\delta L / \lambda)$.

If we consider a photon propagating along one spatial dimension. Suppose we divide its propagation distance $L$ into pieces of $l_{\mathrm{P}}$; within each piece, the phase-shift fluctuation it gains is substantial: $\delta \phi \sim 2 \pi l_{\mathrm{P}} / \lambda$-while fluctuations in different intervals are independent of each other. In this way, the total phase shift of the photon does a "random walk" while the light propagates. At the end of propagation, we have a photon-phase fluctuation of

$$
(\Delta \phi)_{1 \mathrm{D}} \sim \sqrt{N_{L}} \delta \phi \sim \sqrt{l_{\mathrm{P}} L / \lambda^{2}},
$$

which is exactly Eq. (1) with $\alpha=1 / 2$.

However, controversies about the magnitude of this wave-front distortion effect were raised in several papers. Coule [9] first qualitatively argued that this decoherence effect would be very small because of the van CittertZernike theorem [10]. Later, by modeling the discrete 
space-time in a Lorentz-invariance way, Dowker et al. [11] calculated a simple model describing the electromagnetic potential generated by oscillating charges at the source rather than studying the independent dynamics of the light wave field. They found that the signal due to space-time foam would be undetectably tiny.

In our paper, we focus on the independent dynamics of light wave fields in space-time with Planck-scale fluctuations by quantitatively studying a model. Actually the light field, as a wave, does not propagate in the way suggested in [1], but in the following Huygens-Fresnel way: when a photon travels through a space-time region, it does not follow only one particular ray, whose length might be subject to the fundamental "fuzziness" prescribed by Eq. (2), but instead, the wave nature of light, or the quantum mechanical nature of the photon, dictates that the photon would simultaneously sample an ensemble of many different neighboring rays, each of which has a potentially different realization of the fundamental length fluctuation; the actual path-length fluctuation must then be given by an averaging among these different length fluctuations. This allows $\Delta \phi$ to go below $2 \pi \delta L / \lambda$. Moreover, because the linear size of the sampling region can be much bigger than $l_{\mathrm{P}}$ (the correlation length of fundamental quantum fluctuations), this averaging can dramatically suppress the actual $\Delta \phi$ from $2 \pi \delta L / \lambda$, or Eq. (1).

In other (simpler) words: (i) propagation of photons is described by the photons' wave function, which describes electromagnetic (EM) waves; (ii) diffraction of EM waves makes them insensitive to fluctuations and disturbances at scales much less than the wavelength; and (iii) as an EM wave propagates through a large distance, the coherence level of its phase front increases unless further disturbance keeps coming [10] (This point was also independently discussed qualitatively by D. H. Coule [9]). If we divide 3 -D space into cubes of side length $\sim l_{\mathrm{P}}$, with disturbance to light propagation independent within each cube, then using Fourier optics, it is easy to estimate that the effect we have described above will be suppressed by $\sqrt{l_{\mathrm{P}} / \lambda}$ in each transverse direction (because only perturbations with spatial frequencies below $\sim 1 / \lambda$ along each transverse direction get registered by the propagating light), which leads to

$$
\Delta \phi_{3 \mathrm{D}}=\Delta \phi_{1 \mathrm{D}}\left(l_{\mathrm{P}} / \lambda\right) \text {. }
$$

In the following, before discussing the consequences of this rather dramatic suppression, we shall justify Eq. (4) at a very pedagogical level.

\section{MODEL OF SPACE-TIME FLUCTUATION}

Let us construct a toy model reflecting the effect of quantum space-time fluctuation induced length uncertainty on the propagation of light. In this model, the Minkowski space-time with length fluctuation at each point is viewed as a "medium" with a random (yet static) distribution of refractive index $n(x, y, z) \equiv 1+\varepsilon(x, y, z)$. We assume the following translational invariant spatial autocorrelation function for $\varepsilon$ :

$$
\begin{aligned}
& \left\langle\varepsilon\left(x^{\prime}, y^{\prime}, z^{\prime}\right) \varepsilon\left(x^{\prime \prime}, y^{\prime \prime}, z^{\prime \prime}\right)\right\rangle \\
& \quad=a^{2} \Pi\left(x^{\prime}-x^{\prime \prime}\right) \Pi\left(y^{\prime}-y^{\prime \prime}\right) \Pi\left(z^{\prime}-z^{\prime \prime}\right),
\end{aligned}
$$

where " $\langle\ldots\rangle$ " stands for ensemble average, $a$ is of order unity, and

$$
\Pi(x)= \begin{cases}1 & |x| \lesssim l_{\mathrm{P}} \\ 0 & |x| \gg l_{\mathrm{P}}\end{cases}
$$

Our purpose is to study the propagation of light as a scalar wave traveling in this medium. In this specific toy model, the coordinate speed of light propagation fluctuates, in on a small region with the size comparable to the Planck scale-this simulates light propagation in a space-time with Planck-scale quantum fluctuations. In addition, light-speed fluctuations in regions separated by more than the Planck length are independent of each other following the random walk model. As we shall see in the calculation below, the particular shape of the correlation function Eq. (5) does not matter-the effect will remain the same as long as: (i) the total variance in $n$ is of order unity, and (ii) coherence in $\varepsilon$ exists only between points separated by less than $l_{\mathrm{p}}$. (iii) Our toy model entails a quantum gravity induced breaking of Lorentz invariance which has been strongly restricted by astrophysical data [5]. However, our toy model should be sufficient to demonstrate the omitted effect in [1-4] due to the wave nature of light and to capture the key character of the previously proposed idea.

\section{THE WAVE EQUATION}

Returning to the wave picture, we first write down the wave equation:

$$
-[1+2 \varepsilon(x, y, z)] \frac{\partial^{2} \Phi}{\partial t^{2}}+\nabla^{2} \Phi=0 .
$$

Since our refractive-index perturbation is static, we can expand the total wave into two monochromatic pieces, the ideal wave $\Phi_{0}(x, y, z) e^{-i \omega_{0} t}$ and the scattered wave $\psi(x, y, z) e^{-i \omega_{0} t}$.

$$
\Phi=\left[\Phi_{0}(x, y, z)+\psi(x, y, z)\right] e^{-i \omega_{0} t} .
$$

The ideal wave is the unperturbed part of the light field satisfying: $-\partial^{2} \Phi_{0} / \partial t^{2}+\nabla^{2} \Phi_{0}=0$. At leading order in $\varepsilon$, we have

$$
\left(\nabla^{2}+\omega_{0}^{2}\right) \psi(x, y, z)=-2 \omega_{0}^{2} \varepsilon(x, y, z) \Phi_{0}(x, y, z),
$$

which means $\psi$ is a perturbative field sourced by a beat between the ideal wave and space-time perturbations. 
We know from the observed phenomenology that such a perturbation must apply to our situation: namely, fluctuation caused by the space-time foam is indeed very weak compared with an ideal wave propagating across the Universe, and we have $\left|\psi / \Phi_{0}\right| \ll 1$.

For a point source, we assume

$$
\Phi_{0}(x, y, z)=\frac{e^{i \omega_{0} r}}{4 \pi r}, \quad r \equiv \sqrt{x^{2}+y^{2}+z^{2}} .
$$

At the distance $L$, the scattered wave $\psi$ must be compared with the ideal wave to characterize the modulation caused to the ideal spherical wave by space-time perturbations. Let us define

$$
\alpha+i \phi \equiv \frac{\psi}{\Phi_{0}}=4 \pi L \psi e^{-i \omega_{o} L}, \quad \alpha, \phi \in \mathbb{R},
$$

so $\alpha$ describes the amplitude modulation, and $\phi$ describes phase modulation in radians. We also define the total modulation,

$$
\xi \equiv \sqrt{\left\langle\alpha^{2}+\phi^{2}\right\rangle}=4 \pi L \sqrt{\left\langle\psi \psi^{*}\right\rangle},
$$

whose standard deviation is greater than those of both the amplitude and the phase modulations.

\section{SUMMING OVER PATHS}

To arrive at the answer quickly, we use the HuygensFresnel-Kirchhoff scalar diffraction theory, which is equivalent to applying the outgoing Green function, and obtain [12]:

$$
\begin{aligned}
\psi(\mathbf{x}) & =\int_{\left|\mathbf{x}^{\prime}\right|<L}-2 \omega_{0}^{2} \varepsilon\left(\mathbf{x}^{\prime}\right) \Phi_{0}\left(\mathbf{x}^{\prime}\right) \frac{e^{i \omega_{0}\left|\mathbf{x}-\mathbf{x}^{\prime}\right|}}{4 \pi\left|\mathbf{x}-\mathbf{x}^{\prime}\right|} d \mathbf{x}^{\prime} \\
& =\int_{\left|\mathbf{x}^{\prime}\right|<L} \frac{e^{i \omega_{0}\left|\mathbf{x}^{\prime}\right|}}{4 \pi\left|\mathbf{x}^{\prime}\right|}\left[-2 \omega_{0}^{2} \varepsilon\left(\mathbf{x}^{\prime}\right)\right] \frac{e^{i \omega_{0}\left|\mathbf{x}-\mathbf{x}^{\prime}\right|}}{4 \pi\left|\mathbf{x}-\mathbf{x}^{\prime}\right|} d \mathbf{x}^{\prime} .
\end{aligned}
$$

Note that we have considered only contributions from fluctuations at distances smaller than $L$ to the point source. The integral (13) can be interpreted as a path integralover all paths that consist of two straight sections (each associated with a propagator), and a deflection in the middle due to interaction with refractive-index fluctuations (associated with a coupling coefficient). Paths with more than one deflection do not have to be taken into account in our perturbative treatment at leading order.

If we discretize the integration domain, a sphere with volume $\sim L^{3}$, into cells with linear size $\sim l_{\mathrm{P}}$ and volume $v_{\mathrm{P}} \sim l_{\mathrm{P}}^{3}$, we will get a total of $N_{\text {tot }} \sim L^{3} / l_{\mathrm{P}}^{3}$ individual cells, each of which has a statistically independent fluctuation in $\varepsilon$ with variance $a^{2}$ [cf. Eq. (5)]. Then the fluctuation variance of $\psi$ given in Eq. (13) can be estimated in the following way:

$$
\left\langle|\psi|^{2}\right\rangle \sim \omega_{0}^{4} \int d \mathbf{x} d \mathbf{x}^{\prime} \frac{e^{i \omega_{0}\left(\left|\mathbf{x}^{\prime}\right|+\left|\mathbf{x}-\mathbf{x}^{\prime}\right|-\left|\mathbf{x}^{\prime \prime}\right|-\left|\mathbf{x}-\mathbf{x}^{\prime \prime}\right|\right)}}{\left|\mathbf{x}-\mathbf{x}^{\prime \prime}\right|\left|\mathbf{x}-\mathbf{x}^{\prime}\right|\left|\mathbf{x}^{\prime}\right|\left|\mathbf{x}^{\prime \prime}\right|}\left\langle\varepsilon\left(\mathbf{x}^{\prime}\right) \varepsilon\left(\mathbf{x}^{\prime \prime}\right)\right\rangle,
$$

where the integration is over the region $|\mathbf{x}|<L,\left|\mathbf{x}^{\prime}\right|<L$. Since the correlation function of $\varepsilon(\mathbf{x})$ only contributes to the integral when $\left|\mathbf{x}^{\prime}-\mathbf{x}^{\prime \prime}\right| \leq\left|l_{P}\right|$, therefore the exponential in the numerator is approximately equal to 1 . If then we change the integration argument using $\Delta \mathbf{x}=\mathbf{x}^{\prime}-\mathbf{x}^{\prime \prime}$ and $2 \mathbf{x}_{0}=\mathbf{x}^{\prime}+\mathbf{x}^{\prime \prime}$ while substituting Eq. (5), the variance becomes:

$$
\left\langle|\psi|^{2}\right\rangle \sim \omega_{0}^{4} \int_{|\Delta \mathbf{x}|<l_{P}} d^{3} \Delta \mathbf{x} \int_{\left|\mathbf{x}_{0}\right|<L} d^{3} \mathbf{x}_{0} \frac{1}{\left|\mathbf{x}_{0}\right|^{2}\left|\mathbf{x}-\mathbf{x}_{0}\right|^{2}} .
$$

The first integral over $\Delta \mathbf{x}$ is just $l_{P}^{3}$ while the second one over $\mathbf{x}_{0}$ can be approximated to be $L^{3} / L^{4}$. Using the definition of $v_{P}$ and $N_{\text {total }}$, finally we have

$$
\sqrt{\left\langle|\psi|^{2}\right\rangle} \sim \frac{\omega_{0}^{2} a}{L^{2}} v_{\mathrm{P}} \sqrt{N_{\mathrm{tot}}} \sim a \omega_{0}^{2} \sqrt{l_{\mathrm{P}}^{3} / L} .
$$

According to Eq. (12) and comparing with Eq. (3), we have

$$
\Delta \phi \lesssim \xi \sim a \omega_{0}^{2} \sqrt{l_{\mathrm{P}}^{3} L} \sim(\Delta \phi)_{1 \mathrm{D}}\left(l_{\mathrm{P}} / \lambda\right) .
$$

There is an extra suppression factor of $l_{\mathrm{P}} / \lambda$, which arises from the fact that in Eq. (13), the intermediate point $\mathbf{x}^{\prime}$ of the optical path has the freedom to depart away from the axis connecting the source point and the field point, and sample through $N_{\text {tot }} \sim L^{3} / l_{\mathrm{P}}^{3}$ independent fluctuations, instead of only $N_{L} \sim L / l_{\mathrm{P}}$ in the one-dimensional treatment. A more precise calculation gives only an additional numerical factor of the order of unity:

$$
\Delta \phi \lesssim \xi=\sqrt{\frac{\pi}{8}} a \omega_{0}^{2} \sqrt{l_{\mathrm{P}}^{3} L} .
$$

We have therefore confirmed Eq. (4).

\section{SPATIAL-SCALE CUTOFF}

To separately study fluctuations at different spatial scales, we solve the same problem by decomposing the secondary wave into modes:

$$
\psi(r, \theta, \phi)=\sum_{\ell m}\left[\psi_{\ell m}(r) Y_{\ell m}(\theta, \varphi)\right] .
$$

Here $Y_{\ell m}(\theta, \varphi)$ are spherical harmonics. They describe angular variations at scales of $2 \pi / \ell$; at a radius $r$, that corresponds to transverse length scales of $2 \pi r / \ell$, or transverse spatial frequencies of $\ell /(2 \pi r)$. The modal decomposition of Eq. (9) is 


$$
\begin{aligned}
& {\left[\frac{1}{r} \frac{\partial}{\partial r}\left(r \frac{\partial}{\partial r}\right)+\omega_{0}^{2}-\frac{\ell(\ell+1)}{r^{2}}\right] \psi_{\ell m}(r)} \\
& \quad=-\frac{\omega_{0}^{2} e^{i \omega_{0} r} \varepsilon_{\ell m}(r)}{2 \pi r},
\end{aligned}
$$

with

$$
\varepsilon_{\ell m}(r) \equiv \int_{0}^{2 \pi} d \varphi \int_{0}^{\pi} \sin \theta d \theta\left[\varepsilon(r, \theta, \varphi) Y_{\ell m}^{*}(\theta, \varphi)\right]
$$

which satisfies

$$
\left\langle\varepsilon_{\ell m}(r) \varepsilon_{\ell^{\prime} m^{\prime}}^{*}\left(r^{\prime}\right)\right\rangle=a^{2} \delta_{\ell \ell^{\prime}} \delta_{m m^{\prime}} \delta\left(r-r^{\prime}\right) l_{\mathrm{p}}^{3} / r^{2} .
$$

Here we simply assumed

$$
\left\langle\varepsilon(\mathbf{x}) \varepsilon\left(\mathbf{x}^{\prime}\right)\right\rangle=a^{2} l_{\mathrm{P}}^{3} \delta^{(3)}\left(\mathbf{x}-\mathbf{x}^{\prime}\right) .
$$

The spatial spectrum corresponding to this correlation function is identical to that in Eq. (5) at low spatial frequencies, but continues to exist in orders higher than $1 / l_{\mathrm{p}}$. In principle, those modes may also add incoherently to our output fluctuations, but as we shall see, their contributions will be negligible.

Solving Eq. (20), assuming regularity at $r=0$ and the outgoing wave condition at $r=L$, we obtain:

$$
\psi_{\ell m}(L)=-\frac{\omega_{0}^{3} L h_{\ell}^{(1)}\left(\omega_{0} L\right)}{2 \pi} \int_{0}^{L} d r\left[r j_{\ell}\left(\omega_{0} r\right) e^{i \omega_{0} r} \varepsilon_{\ell m}(r)\right],
$$

where $j_{\ell}$ and $h_{\ell}^{(1)}$ are spherical Bessel and first-kind spherical Hankel functions [13]. From Eqs. (22) and (24), we obtain

$$
16 \pi^{2} L^{2}\left\langle\psi_{\ell m} \psi_{\ell^{\prime} m^{\prime}}^{*}\right\rangle=\xi_{\ell m}^{2} \delta_{\ell \ell^{\prime}} \delta_{m m^{\prime}}
$$

with

$\xi_{\ell m}^{2} \equiv 4 a^{2}\left|\omega_{0} L h_{\ell}^{(1)}(L)\right|^{2}\left(\omega_{0} l_{\mathrm{P}}\right)^{3} \int_{0}^{L} j_{\ell}^{2}\left(\omega_{0} r\right) \omega_{0} d r$.

Note that $\xi_{\ell m}$ is independent of $m$, which is a consequence of the rotation invariance of the refractive-index fluctuations. The total fluctuation at $r=L$ will then be

$$
\xi^{2}=\sum_{\ell=0}^{+\infty} \frac{2 \ell+1}{4 \pi} \xi_{\ell 0}^{2} \equiv \sum_{\ell=0}^{+\infty} \xi_{\ell}^{2} .
$$

Physically, $\xi_{\ell}^{2}$ describes fluctuations at the angular scale $\sim 2 \pi / \ell$, or transverse spatial scales $2 \pi L / \ell$, or transverse spatial frequency of $\ell /(2 \pi L)$. Inserting Eq. (26), we have

$$
\begin{aligned}
\xi_{\ell}^{2}= & a^{2}\left(\omega_{0} l_{\mathrm{P}}\right)^{3} \frac{2 \ell+1}{\pi}\left|\omega_{0} L h_{\ell}^{(1)}\left(\omega_{0} L\right)\right|^{2} \\
& \times \int_{0}^{\omega_{0} L} j_{\ell}^{2}(R) d R .
\end{aligned}
$$

We expect $\ell \sim \omega_{0} L$, or $2 \pi L / \ell \sim \lambda$ to be the turning point, because at this point the transverse spatial scale is comparable to the wavelength $\lambda$.

Mathematically, for $\ell<\omega_{0} L$, the spherical Bessel and Hankel functions are wavelike at $r \sim L$, indicating propagating waves; for $\ell>\omega_{0} L$, the spherical Bessel and Hankel functions are not wavelike at $r \sim L$, indicating evanescent waves. In the limiting regimes of $\ell \ll \omega_{0} L$ and $\ell \gg \omega_{0} L, \xi_{\ell}$ can be evaluated analytically, using asymptotic expansions of spherical Bessel functions:

$$
\frac{\xi_{\ell}^{2}}{a^{2}\left(\omega_{0} l_{\mathrm{P}}\right)^{3}}= \begin{cases}1 / 2, & \ell \ll \omega_{0} L, \\ \omega_{0} L /\left[(2 \ell+1)^{2} \pi\right], & \ell \gg \omega_{0} L .\end{cases}
$$

Note that not only does $\xi_{\ell}^{2}$ approach 0 at orders $\ell \gg \omega_{0} L$, the summation of all these higher modes also gives a negligible contribution. This qualitatively confirms a cut-off at the transverse scale of $\lambda$ : fluctuations at much finer scales do not generate a secondary wave. This justifies our original use of Eq. (23), and also ensures that the detailed shape of the correlation function (5) does not matter. In Fig. 1, we study the transition zone of $\ell \sim \omega_{0} L$ numerically, for moderately large values of $\omega_{0} L=100$, 200 , and 300 , by plotting $\xi_{\ell}^{2}$ as a function of $\ell /\left(\omega_{0} L\right)$. As $\omega_{0} L \rightarrow+\infty, \xi_{\ell}^{2}$ asymptotes to a smooth, nonzero function for $\ell /\left(\omega_{0} L\right)<1$, and to 0 for $\ell /\left(\omega_{0} L\right)>1$. This means, in the realistic situation of $\omega_{0} L \ll 1, \ell /\left(\omega_{0} L\right)=1$ is a sharp turning point between propagating and evanescent waves.

It might seem difficult to evaluate the summation (28) analytically. But since we are solving exactly the same problem as the previous section, it should be clear that [cf. Eq. (18)]

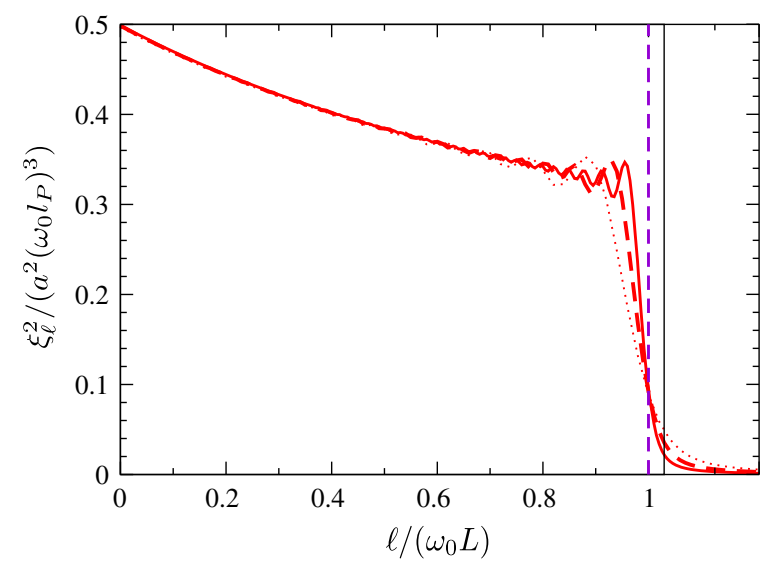

FIG. 1 (color online). Plots of $\xi_{\ell}^{2}$ as functions of $\ell /\left(\omega_{0} L\right)$, for cases with $\omega_{0} L=100$ (dotted curve), 200 (dashed curve), and 300 (solid curve). 


$$
\xi=\sqrt{\sum_{\ell=0}^{+\infty} \xi_{\ell}^{2}}=\sqrt{\frac{\pi}{8}} a \omega_{0}^{2} \sqrt{l_{\mathrm{P}}^{3} L} \sim(\Delta \phi)_{1 \mathrm{D}}\left(l_{\mathrm{P}} / \lambda\right),
$$

as we have verified numerically in the cases $\omega_{0} L=100$, 200 , and 300 .

\section{DISCUSSIONS}

So far in this paper, we have calculated fluctuations on the phase front of an extragalactic point source, caused by Planck-scale fluctuations in refractive index - a toy model for space-time foam. If diffraction of light were ignored, or if we assumed a space-time with one time dimension plus one spatial dimension, our toy model would give comparable results to previous estimates on the random-walk model claimed in [1-4]. However, the diffraction of light requires us to average space-time fluctuations over all different possible optical paths that extend to all three spatial dimensions. This averaging filters out all fluctuations with transverse scales finer than the wavelength. In our model, this causes an extra suppression factor of $l_{\mathrm{P}} / \lambda$, with [cf. Eq. (1)]

$$
\Delta \phi \lesssim \sqrt{l_{\mathrm{P}} L / \lambda^{2}}\left(l_{\mathrm{P}} / \lambda\right)
$$

With respect to previous literature, this suppression is at least by 29 orders of magnitude for astronomical observations, if the wavelength of $\lambda=10^{-6} \mathrm{~m}$ were to be used. Numerically, we have

$$
\Delta \phi \sim 10^{-26} \sqrt{L / G p c}\left(5 \times 10^{-7} \mathrm{~m} / \lambda\right)^{2},
$$

which makes the quantum foam effect on the light propagation extremely small. Suppose we consider another type of experiment, namely the use of $\gamma$-ray arrival time. It is straightforward to convert

$$
\Delta t=\Delta \phi \frac{\lambda}{c}=t_{P} \sqrt{l_{\mathrm{P}} L / \lambda^{2}}=10^{-33} \mathrm{~s} \sqrt{L / \mathrm{Gpc}}\left(E_{\gamma} / \mathrm{GeV}\right) .
$$

For both methods, the effect of random-walk-type spacetime foam is too small to be seen.

For certain extra-dimension models [14], e.g., the one raised by Arkani-Hamed et al. [15], in which the fundamental scale of nature is the electroweak scale $l_{\mathrm{EM}} \sim 10^{-18} \mathrm{~m}, l_{\mathrm{P}}$ is amplified by a large factor, therefore apparently increasing the detectability of quantum foams. Using $l_{\mathrm{P}} \rightarrow l_{\mathrm{EM}} \sim 10^{-18} \mathrm{~m}$, we update the above estimates to

$$
\Delta \phi_{\mathrm{ed}} \sim 0.1 \sqrt{L / \mathrm{Gpc}}\left[\left(5 \times 10^{-7} \mathrm{~m}\right) / \lambda\right]^{2},
$$

and

$$
\Delta t_{\mathrm{ed}} \sim\left(10^{-8} \mathrm{~s}\right) \sqrt{L / \mathrm{Gpc}}\left(E_{\gamma} / \mathrm{GeV}\right) .
$$

At first sight, these seem more promising to detect. However, we must be more careful in connecting $\Delta \phi_{\text {ed }}$ and $\Delta t_{\mathrm{ed}}$, which are the total variance after summing over all spatial frequencies, to observables in actual experiments. In both cases, from our study of wave propagation, we have

$$
\left\langle\Delta \phi(\vec{x}) \Delta \phi\left(\vec{x}^{\prime}\right)\right\rangle \approx \begin{cases}\Delta \phi_{\mathrm{ed}}^{2} & \left|\vec{x}-\vec{x}^{\prime}\right| \lesssim \lambda \\ 0 & \left|\vec{x}-\vec{x}^{\prime}\right|>\lambda\end{cases}
$$

and a similar relation for the two-point correlation function of $\Delta t(\vec{x})$.

For a telescope image, the ideal wave gives an Airy pattern on the focal plane, while the scattered wave would create a diffuse background on the focal plane, which has a total energy of $\Delta \phi_{\text {ed }}^{2}$, and therefore a flux $\mathscr{F}_{\text {background }}^{P l}$ that is

$$
\mathscr{F}_{\text {background }}^{P l} / \mathscr{F}_{\text {image }} \sim \Delta \phi_{\text {ed }}^{2}\left(\frac{\lambda^{2}}{\mathscr{A}}\right),
$$

compared with the typical flux of the ideal image. It could be almost impossible to detect such a background due to confusion with other types of background. For example, we can estimate the magnitude of (37) for a typical quasar source PG2112 + 059, which is $\sim 2.5 \mathrm{Gpc}$ away from us. According to the observational data collected by using the Hubble Space Telescope (Wide Field Camera 3), at wavelength $\lambda \sim 1.25 \times 10^{-6} \mathrm{~m}$, the ratio between photon flux of the sky background and photon flux of the image is $\sim 0.7 \times 10^{-3}$ [16]. However, $\mathscr{F}_{\text {background }}^{P l} / \mathscr{F}_{\text {image }}$ is $\sim 0.8 \times 10^{-15}$, which is $10^{12}$ smaller.

For detecting arrival time of $\gamma$-ray photons, we have to be aware that the true $\Delta t$ also depends on the averaging area of our detector-which corresponds to the pixel size, which we also denote by $\mathscr{A}$. As a consequence, we have

$$
\Delta t_{\mathrm{obs}}=\Delta t_{\mathrm{ed}} \sqrt{\frac{\lambda^{2}}{\mathscr{A}}},
$$

which is likely to gain an additional factor from $\Delta t_{\mathrm{ed}}$. The actual time resolution of a gamma ray detector, e.g., Fermi Telescope, is $\sim 10 \mu \mathrm{s}$ [17]. From (36), the observed correction to the arrival time of gamma ray photons with $E_{\gamma}=1 \mathrm{GeV}$ and source distance $\sim 1 \mathrm{Gpc}$ is $\sim 10^{-23} \mathrm{~s}$, which is also too small to resolve.

What has been left out in this paper is possible fluctuation in time. If the refractive index fluctuation has a white noise spectrum up to the Planck frequency, then by observing a portion of the optical spectrum $\Delta \omega$, the effect will be suppressed further by a factor of

$$
\sqrt{t_{\mathrm{P}} \Delta \omega}
$$

which is likely to be very large as well. 


\section{ACKNOWLEDGMENTS}

The authors would like to thank D. G. Blair, Z. Cai, X. Fan, H. Nikolai, and C. Zhao for helpful discussions. We thank Ning Jiang for this help in calculating the background of quasar PG2112 + 059. Y. C. and L. W. were supported by the Alexander von Humboldt Foundation's Sofja Kovalevskaja Programme (funded by the German
Federal Ministry of Education and Research). We also thank Dr. Ron Burman for reading the manuscript. Y. C. is currently supported by NSF Grant No. PHY-1068881 and CAREER Grant No. PHY-0956189. L. W. acknowledges funding supports from the Australian Research Council. Y. M. is currently supported by the Australian Department of Education, Science and Training.
[1] Y. J. Ng, W. A. Christiansen, and H. van Dam, Astrophys. J. 591, L87 (2003).

[2] R. Lieu and L. W. Hillman, Astrophys. J. 585, L77 (2003).

[3] R. Ragazzoni, M. Turatto, and W. Gaessler, Astrophys. J. 587, L1 (2003).

[4] W. A. Christiansen, Y. J. Ng, and H. van Dam, Phys. Rev. Lett. 96, 051301 (2006).

[5] G. Amelino-Camelia, Living Rev. Relativity 16, 5 (2013).

[6] S. Lloyd and Y. J. Ng, Sci. Am. 291, 52 (2004).

[7] V. Giovannetti, S. Lloyd, and L. Maccone, Science 306, 1330 (2004).

[8] Michael Maziashvili, arXiv:hep-ph/0605146.

[9] D. H. Coule, Classical Quantum Gravity 20, 3107 (2003).

[10] http://en.wikipedia.org/wiki/Van_CittertZernike_theorem.

[11] F. Dowker, J. Henson, and R. D. Sorkin, Phys. Rev. D 82, 104048 (2010).
[12] J. D. Jackson, Classical Electrodynamics, 3rd ed. (John Wiley \& Sons, New York, 1999), Sec. 10.5.

[13] In order to obtain Eq. (24), we break the right-hand side of Eq. (20) into an integral over $\delta$-functions: $\int d r^{\prime} F(r) \delta\left(r-r^{\prime}\right)$. For each $\delta\left(r-r^{\prime}\right)$, its contribution must be of the form $A j_{\ell}\left(\omega_{0} r\right)$ for $r<r^{\prime}$ (due to regularity at origin) and $B h_{\ell}^{(1)}\left(\omega_{0} r\right)$ for $r>r^{\prime}$ (due to outgoing condition at infinity). Then $A$ and $B$ can be solved by applying continuity in $\psi$, and junction condition in $\partial \psi / \partial r$.

[14] R. Maartens and K. Koyama, Living Rev. Relativity 13, 5 (2010).

[15] N. Arkani-Hamed, S. Dimopoulos, and G. Dvali, Phys. Lett. B 429, 263 (1998).

[16] Ning Jiang (private communication).

[17] Fermi LAT Performance, http://www.slac.stanford.edu/exp/ glast/groups/canda/lat_Performance.htm. 